Paper

\title{
Generalized synchronization and complexity in unidirectionally coupled dynamical systems
}

\author{
Kazutaka Kanno ${ }^{1 a)}$ and Atsushi Uchida $\left.{ }^{1 b}\right)$ \\ ${ }^{1}$ Department of Information and Computer Sciences, Saitama University, \\ 255 Simo-okubo, Sakura-ku, Saitama City, Saitama 338-8570, Japan \\ a) s11dm001@mail.saitama-u.ac.jp \\ b) auchida@mail.saitama-u.ac.jp
}

Received August 22, 2011; Revised December 5, 2011; Published April 1, 2012

\begin{abstract}
We investigate generalized synchronization and complexity in unidirectionally coupled Rössler systems and Mackey-Glass systems with time-delayed feedback. The generalized synchronization is measured by using two identical response systems which are driven by a common drive system. To measure the complexity, KS entropy and KY dimension are calculated quantitatively from the Lyapunov spectrum by using the linearized equations. The response systems show generalized synchronization for large coupling strengths. The entropy and dimensionality decrease as the coupling strength is increased. The minimum value of entropy is obtained at the condition of generalized synchronization, whose value corresponds to the entropy for the solitary drive system, even though the temporal waveforms of the drive and response systems are significantly different. The maximum entropy is achieved outside the region where generalized synchronization is achieved, whose value almost corresponds to the sum of those of the two solitary drive and response systems. It is found that the coupling effect changes the entropy within these two values. The dimensionality decreases monotonically even within the region of generalized synchronization.
\end{abstract}

Key Words: consistency, synchronization, complexity, Lyapunov spectrum, Rössler model, Mackey-Glass model

\section{Introduction}

Generalized synchronization has been studied in various dynamical systems, and may have important applications in methods for noninvasive testing and monitoring of structures and materials [1-5]. When a signal from one system (drive) drives another system (response), there is a functional relation between the dynamics of the drive and response system for generalized synchronization. When replicas or duplicates of the response system are available, the auxiliary system method has been suggested for detecting generalized synchronization [1]. Two identical response systems are coupled with the drive system in the auxiliary system approach. If the response systems, starting from different initial conditions, display identical synchronization after transients have disappeared, the response signal is 
generally synchronized to the drive.

It has been known that many nonlinear dynamical systems have an ability to generate consistent outputs when driven by a repeated external signal. This phenomenon is referred to as consistency $[6$, 7] or reliability $[8,9]$, which is defined as the reproducibility of response waveforms in a nonlinear dynamical system driven repeatedly by a signal, starting from different initial conditions of the system. The viewpoint of consistency or reliability is based on the reproducibility of a single response system with respect to a complex drive signal, which could be useful when a dynamical system cannot be made a copy. On the contrary, the viewpoint of generalized synchronization is based on the temporal relationship among coupled multiple dynamical systems. The conditions for achieving consistency and generalized synchronization can be evaluated by using the maximum conditional Lyapunov exponent.

Consistency of response has been experimentally observed in a solid-state laser [6]. The concept of consistency could be applied for an implementation of physical one-way function [10], where an output signal can be easily produced from an input signal through a complex function, whereas the input signal cannot be estimated from the output signal. The physical implementation of one-way function has been reported with a token with complex speckle scattering patterns of light [10]. Instead of using spatial complex patterns, temporal dynamics may be useful when a dynamical system has consistent response. The use of consistency may lead to a new technique of the implementation of physical one-way function, which could be a key technique for hardware-oriented information security systems.

One of the important characteristics of physical one-way function is the complexity of the functional system. The complexity of a single semiconductor laser with time-delayed optical feedback has been reported [11]. Recently, we have numerically investigated consistency and complexity in unidirectionally coupled semiconductor lasers with time-delayed optical feedback [12]. The complexity of the coupled semiconductor lasers can be estimated quantitatively by using the Kolmogorov-Sinai (KS) entropy and the Kaplan-Yorke (KY) dimension, which are derived from the Lyapunov spectrum. It is found that the complexity of the coupled laser system strongly depends on the degree of consistency. The complexity becomes the minimum value under the condition of consistency in the coupled lasers. On the other hand, the maximum value of the complexity can be obtained, which is larger than the sum of the complexity between the solitary drive and response lasers without optical coupling. Therefore, the optical coupling results in significant increase of the complexity in the coupled semiconductor lasers.

It has not been clearly understood that how the optical coupling increases the complexity of coupled dynamical laser systems, and whether this is a universal phenomenon for coupled dynamical systems. In order to investigate the relationship between consistency (or generalized synchronization) and the complexity, it is very important to use more general nonlinear dynamical models, such as the Rössler model [13]. The relationship between generalized synchronization and dimensionality has been investigated in coupled Rössler-Lorenz models [14], although the complexity has not been investigated. In addition, a general model with time-delayed feedback, such as the Mackey-Glass model [15], needs to be used to understand the complexity of the time-delayed laser system.

In this study we investigate the region where generalized synchronization is achieved in unidirectionally coupled nonlinear dynamical systems and measure the complexity of the coupled systems by using the Lyapunov spectrum analysis. We use the coupled Rössler models as well as the coupled Mackey-Glass models with time-delayed feedback for the investigation of the relationship between generalized synchronization and complexity. We quantitatively evaluate the complexity of the coupled dynamical systems by using the KS entropy and the KY dimension, which can be estimated from the Lyapunov spectrum.

\section{Models}

To investigate the parameter region where generalized synchronization is achieved, we use a model consisting of three dynamical systems, known as auxiliary system approach [1], as shown in Fig. 1. Response 1 and 2 systems have identical parameter values, but different initial conditions. There is no coupling between the response 1 and 2. A signal from a drive system is simultaneously input into 


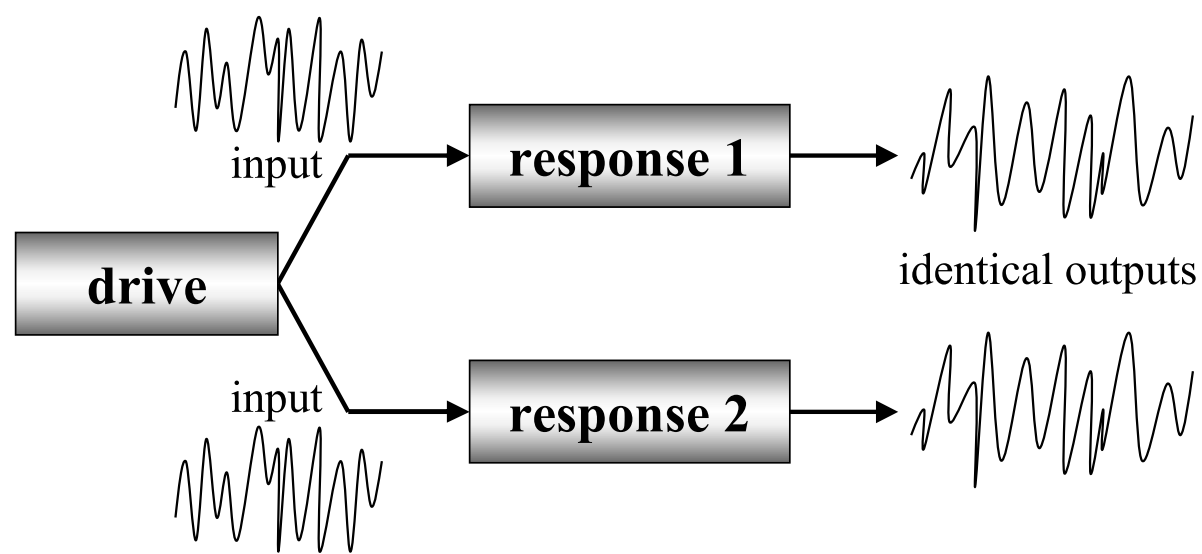

Fig. 1. Schematic of generalized synchronization in three coupled dynamical systems. Two identical response systems are driven by a signal from a drive system (called drive, response 1 , and response 2 ).

the response 1 and 2 systems. We consider that generalized synchronization is achieved when similar temporal waveforms are obtained between the response 1 and 2 that are driven by the common drive signal.

\subsection{Coupled Rössler model}

The first example of a coupled dynamical system consists of three coupled Rössler models as follows.

$$
\begin{aligned}
\dot{x}_{d} & =-y_{d}-z_{d} \\
\dot{y}_{d} & =x_{d}+a_{d} y_{d} \\
\dot{z}_{d} & =b_{d}+\left(x_{d}-c_{d}\right) z_{d} \\
\dot{x}_{r 1} & =-y_{r 1}-z_{r 1}+\kappa\left(x_{d}-x_{r 1}\right) \\
\dot{y}_{r 1} & =x_{r 1}+a_{r 1} y_{r 1} \\
\dot{z}_{r 1} & =b_{r 1}+\left(x_{r 1}-c_{r 1}\right) z_{r 1} \\
\dot{x}_{r 2} & =-y_{r 2}-z_{r 2}+\kappa\left(x_{d}-x_{r 2}\right) \\
\dot{y}_{r 2} & =x_{r 2}+a_{r 2} y_{r 2} \\
\dot{z}_{r 2} & =b_{r 2}+\left(x_{r 2}-c_{r 2}\right) z_{r 2}
\end{aligned}
$$

Where, $d, r 1$, and $r 2$ represent drive, response 1, and response 2, respectively. The last terms of the right-hand side in Eqs. (4) and (7) represent unidirectional couplings, where $\kappa$ is the coupling strength between the drive and response systems. We set the parameter values in the following: $a_{d}=a_{r 1}=a_{r 2}=0.2, b_{d}=b_{r 1}=b_{r 2}=0.2, c_{d}=5.7$, and $c_{r 1}=c_{r 2}=7.0$. The response 1 and 2 have identical parameters, whereas one parameter value is different between the drive and response systems $\left(c_{d} \neq c_{r 1}=c_{r 2}\right)$.

\subsection{Coupled Mackey-Glass model}

The second example of a coupled dynamical system consists of three Mackey-Glass models with timedelayed feedbacks as follows.

$$
\begin{gathered}
\dot{x}_{d}=\frac{a_{d} x_{\tau, d}}{1+\left(x_{\tau, d}\right)^{b_{d}}}-c_{d} x_{d} \\
\dot{x}_{r 1}=\frac{a_{r 1} x_{\tau, r 1}}{1+\left(x_{\tau, r 1}\right)^{b_{r 1}}}-c_{r 1} x_{r 1}+\kappa\left(x_{d}-x_{r 1}\right)
\end{gathered}
$$




$$
\dot{x}_{r 2}=\frac{a_{r 2} x_{\tau, r 2}}{1+x_{\tau, r 2}^{b_{r 2}}}-c_{r 2} x_{r 2}+\kappa\left(x_{d}-x_{r 2}\right)
$$

As in the case of Eqs. (1)-(9), $d, r 1$, and $r 2$ represent drive, response 1, and response 2, respectively. $x_{\tau, d}=x_{d}(t-\tau), x_{\tau, r 1}=x_{r 1}(t-\tau)$, and $x_{\tau, r 2}=x_{r 2}(t-\tau)$ are the time-delayed variables, where $\tau$ is the delay time $(\tau=5)$. The last terms of the right-hand side in Eqs. (11) and (12) represent unidirectional couplings, where $\kappa$ is the coupling strength between the drive and response systems. We set the parameter values in the following: $a_{d}=a_{r 1}=a_{r 2}=2.0, b_{d}=b_{r 1}=b_{r 2}=10, c_{d}=1.0$, and $c_{r 1}=c_{r 2}=0.9$.

\section{Lyapunov spectrum analysis}

\subsection{Coupled Rössler model}

Lyapunov exponents, which indicate growth rates of perturbations applied to a trajectory of a dynamical system in the phase space, can be used to quantify the complexity of coupled dynamical systems. To calculate the Lyapunov exponents, linearized equations can be derived from the original dynamical equations,

$$
\dot{\boldsymbol{\delta}}_{x}=D_{x} F\left(\boldsymbol{\delta}_{x}, x\right) \boldsymbol{\delta}_{x}
$$

where $\boldsymbol{\delta}_{x}$ are linearized variables of $x$, and $D_{x} F$ is the Jacobian matrix with respect to $x$. The linearized equations for coupled Rössler system of Eqs. (1)-(6) can be obtained,

$$
\begin{gathered}
\dot{\delta}_{x, d}=-\delta_{y, d}-\delta_{z, d} \\
\dot{\delta}_{y, d}=\delta_{x, d}+a_{d} \delta_{y, d} \\
\dot{\delta}_{z, d}=z_{d} \delta_{x, d}+\left(x_{d}-c_{d}\right) \delta_{z, d} \\
\dot{\delta}_{x, r}=-\delta_{y, r}-\delta_{z, r}+\kappa\left(\delta_{x, d}-\delta_{x, r}\right) \\
\dot{\delta}_{y, r}=\delta_{x, r}+a_{r} \delta_{y, r} \\
\dot{\delta}_{z, r}=z_{r} \delta_{x, r}+\left(x_{r}-c_{r}\right) \delta_{z, r}
\end{gathered}
$$

Equations (14)-(16) and Eqs. (17)-(19) correspond to the linearized equations for the drive and response systems, respectively. The subscript $r 1$ is replaced with $r$ in Eqs. (17)-(19). We measure the complexity of the entire coupled systems (i.e., both the drive and response systems), and it is expected to have more complexity of the entire coupled systems than the solitary system.

The evolution of the linearized variables is calculated and a norm $d$ of all the linearized variables can be obtained as follows,

$$
d(t)=\sqrt{\delta_{x, d}^{2}(t)+\delta_{y, d}^{2}(t)+\delta_{z, d}^{2}(t)+\delta_{x, r}^{2}(t)+\delta_{y, r}^{2}(t)+\delta_{z, r}^{2}(t)} .
$$

From the time evolution of the norm $d$, the maximum Lyapunov exponent $\lambda_{\max }$ can be calculated as follows,

$$
\lambda_{\max }=\frac{1}{h N} \sum_{i=0}^{N-1} \ln \frac{d(t+i h)}{d(t+(i-1) h)},
$$

where $N$ is the number of calculation step, $h$ is the time step for numerical simulation, and ln represents the natural logarithm. $N$ needs to be large enough to converge the value of $\lambda_{\max }$ in numerical simulation. For the calculation of the norm $d$, the linearized variables need to be normalized for each time step $h$ to maintain small perturbation from the chaotic trajectory.

\subsection{Coupled Mackey-Glass model}

For the time-delayed dynamical systems such as the Mackey-Glass model, the linearized equations contain time-delayed variables, and Eq. (13) is written as follows,

$$
\dot{\boldsymbol{\delta}}_{x}=D_{x} F\left(\boldsymbol{\delta}_{x}, x, x_{\tau}\right) \boldsymbol{\delta}_{x}+D_{\tau, x} F\left(\boldsymbol{\delta}_{\tau, x}, x, x_{\tau}\right) \boldsymbol{\delta}_{\tau, x}
$$


where $\boldsymbol{\delta}_{\tau}$ is the time-delayed linearized variables, and $D_{\tau, x} F$ is the Jacobian matrix with respect to $x_{\tau}$. The linearized equations of the unidirectionally coupled Mackey-Glass models with time-delayed feedback can be described as follows,

$$
\begin{gathered}
\dot{\delta}_{x, d}=\frac{a_{d}+a_{d}\left(1-b_{d}\right)\left(x_{\tau, d}\right)^{b_{d}}}{\left[1+\left(x_{\tau, d}\right)^{b_{d}}\right]^{2}} \delta_{\tau, x, d}-c_{d} \delta_{x, d} \\
\dot{\delta}_{x, r}=\frac{a_{r}+a_{r}\left(1-b_{r}\right)\left(x_{\tau, r}\right)^{b_{r}}}{\left[1+\left(x_{\tau, r}\right)^{b_{r}}\right]^{2}} \delta_{\tau, x, r}-c_{r} \delta_{x, r}+\kappa\left(\delta_{x, d}-\delta_{x, r}\right)
\end{gathered}
$$

To calculate the maximum Lyapunov exponent, all the linearized variables included in the delay time $\tau$ need to be regarded as independent variables for the calculation of the norm $d[11,16,17]$.

$$
d(t)=\sqrt{\sum_{i=1}^{M}\left\{\delta_{x, d}(t-\tau+i h)+\delta_{x, r}(t-\tau+i h)\right\}},
$$

where $M$ is the number of state variables discretized by the time step $h$ in the time-delayed loop, which is defined as $M=\tau / h$. The maximum Lyapunov exponent $\lambda_{\max }$ can be obtained as follows,

$$
\lambda_{\max }=\frac{1}{\tau N} \sum_{i=0}^{N-1} \ln \frac{d(t+i \tau)}{d(t+(i-1) \tau)} .
$$

\subsection{Lyapunov spectrum, Kolmogorov-Sinai entropy, and Kaplan-Yorke dimen- sion}

For multi-dimensional nonlinear dynamical systems, the same number of Lyapunov exponents exists as the dimensionality, which are called Lyapunov spectrum. It is necessary to use a set of the linearized equations to calculate Lyapunov spectrum. Each Lyapunov exponent can be obtained from each set of the linearized variables by using orthogonalization of the vector of the linearized variables.

Kolmogorov-Sinai (KS) entropy, which represents unpredictability of dynamical systems, can be obtained from the Lyapunov spectrum. The upper limit of KS entropy $h_{K S}$ can be calculated from the sum of positive Lyapunov exponents.

$$
h_{K S}=\sum_{i=1} \lambda_{i},\left(\lambda_{i}>0\right)
$$

KS entropy indicates a loss rate of information. Larger KS entropy indicates that the dynamical system has larger unpredictability.

Kaplan-Yorke (KY) dimension (also known as Lyapunov dimension), which represents dimensionality of a dynamical system, can be also estimated from the Lyapunov spectrum. KY dimension $D_{K Y}$ can be calculated as follows,

$$
D_{K Y}=j+\frac{\sum_{i=1}^{j} \lambda_{i}}{\lambda_{j+1}}
$$

where $j$ satisfies the following relationship,

$$
\sum_{i=1}^{j} \lambda_{i}<0<\sum_{i=1}^{j+1} \lambda_{i}
$$

KY dimension indicates the number of variables to represent a dynamical system. Larger KY dimension corresponds to more complex dynamics of the dynamical system.

\section{Numerical results of the coupled Rössler model}

\subsection{Observation of generalized synchronization}

First we observe generalized synchronization in the coupled Rössler systems. Figure 2 shows the temporal waveforms of the variables $x$ of the three coupled Rössler systems without or with coupling 

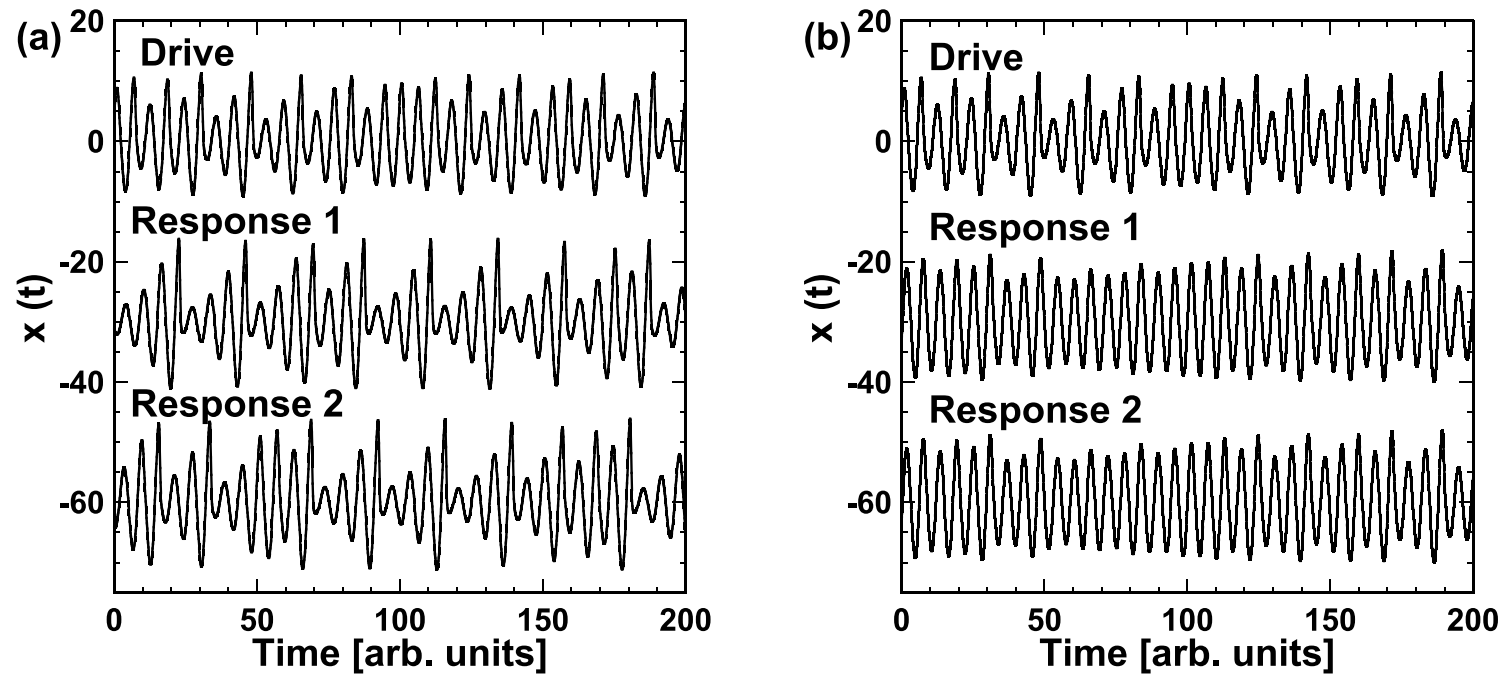

Fig. 2. Temporal waveforms of the drive, response 1 , and response 2 in the coupled Rössler systems (a) without and (b) with coupling between the drive and response systems. The coupling strengths are (a) $\kappa=0$ and (b) $\kappa=0.2$.

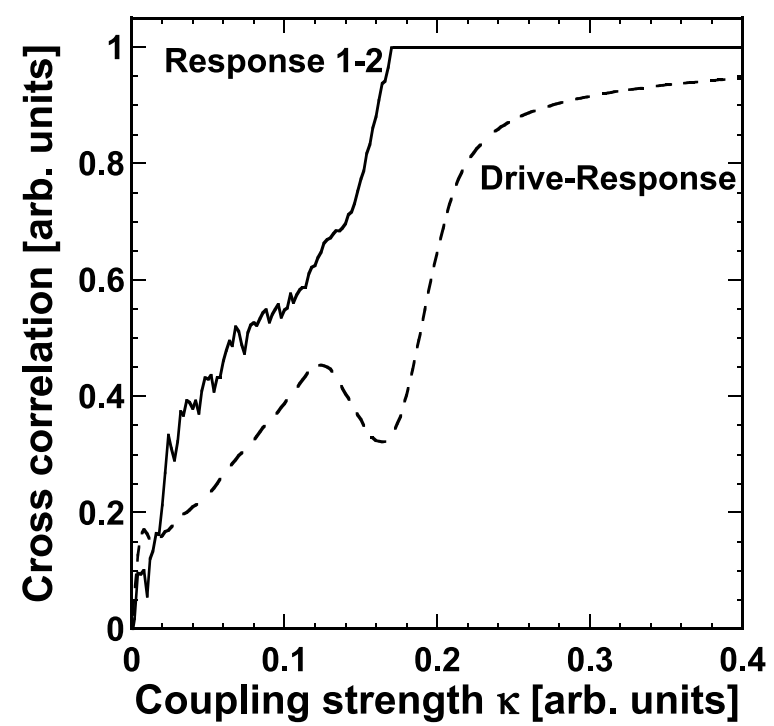

Fig. 3. Cross correlation values $C$ between the response 1 and 2 (solid curve), and between the drive and response 1 (dotted curve) as a function of the coupling strength $\kappa$ in the coupled Rösser systems.

between the drive and response systems. The temporal waveforms of the response 1 and 2 are different without coupling $(\kappa=0)$ and generalized synchronization is not observed as shown in Fig. 2(a). By contrast, the temporal waveforms of the response 1 and 2 are identical at large coupling strength $(\kappa=0.2)$ and generalized synchronization is observed as shown in Fig. 2(b). It is found that generalized synchronization can be achieved by increasing the coupling strength $\kappa$.

We quantitatively evaluate the degree of generalized synchronization by using the cross-correlation function as follows,

$$
C=\frac{<\left(x_{i}-\bar{x}_{i}\right)\left(x_{j}-\bar{x}_{j}\right)>}{\sigma_{i} \sigma_{j}}
$$

where $i$ and $j$ correspond to drive, response 1 , or response 2 system, respectively. $\bar{x}$ and $\sigma$ are the mean value and the standard deviation of the temporal waveform $x$. The angle brackets denote time averaging. When $i$ and $j$ are the response 1 and 2, Eq. (30) represents the cross-correlation between the response 1 and 2 , and $C=1$ indicates the achievement of generalized synchronization.

Figure 3 shows the cross-correlation values as a function of the coupling strength $\kappa$. The solid and dotted curves represent the cross-correlation value between the response 1 and 2 , and that between 
the drive and response 1 , respectively. The cross-correlation between the response 1 and 2 increases with the increase of the coupling strength $\kappa$. The cross correlation value becomes $C=1$ at the region of $\kappa>0.17$, indicating the achievement of generalized synchronization. On the other hand, the crosscorrelation between the drive and response 1 remains $\sim 0.6$ for $\kappa \sim 0.2$, where the temporal waveforms are different between the drive and response systems. It is found that generalized synchronization can be achieved between the response 1 and 2 even though the cross-correlation between the drive and response is relatively low.

\subsection{Generalized synchronization and complexity}

To quantify the complexity of the coupled Rössler systems, we calculate KS entropy $\left(h_{K S}\right)$, and KY dimension $\left(D_{K Y}\right)$ from the Lyapunov spectrum by using a set of the linearized equations. Figure 4(a) shows KS entropy (solid curve) and KY dimension (dotted curve) as a function of the coupling strength $\kappa$. Without coupling $(\kappa=0)$, the value of $h_{K S}$ is 0.150 , corresponding to the sum of $h_{K S}$ for the solitary drive and response systems. As $\kappa$ is increased, $h_{K S}$ slightly decreases and increases, and monotonically decreases at $\kappa>0.04$. $h_{K S}$ becomes almost constant at the region of $\kappa>0.17$, where generalized synchronization is achieved as shown in Fig. 3. This indicates that $h_{K S}$ becomes a minimum value $\left(h_{K S}=0.072\right)$ when generalized synchronization is achieved. This minimum value of the entropy corresponds to the value for the solitary drive system, even though the temporal waveforms of the drive and response systems are significantly different as shown in Fig. 3. On the other hand, the maximum value of $h_{K S}(=0.155)$ can be obtained at $\kappa=0.017$, which is similar to the value of $h_{K S}(=0.150)$ without coupling. Therefore, the entropy cannot dramatically increase in the presence of the coupling, and decreases as $\kappa$ is increased and saturates at large $\kappa$ under the condition of generalized synchronization. For the dimensionality, $D_{K Y}$ is almost constant as $\kappa$ is increased in the region where generalized synchronization is not achieved. However, the dimensionality decreases gradually within the region of generalized synchronization. The entropy and the dimensionality have different characteristics as $\kappa$ is changed.

To interpret the result of Fig. 4(a), we plot the Lyapunov exponents of the coupled Rössler systems as a function of $\kappa$, as shown in Fig. 4(b). Four of the six Lyapunov exponents $\left(\lambda_{1} \sim \lambda_{4}\right.$, where $\left.\lambda_{i} \geq \lambda_{j}(i<j)\right)$ are shown in Fig. 4(b), since the other two Lyapunov exponents are negative with large absolute values $\left(\lambda_{5}=-5.39\right.$ and $\lambda_{6}=-6.69$ at $\left.\kappa=0\right)$. As the coupling strength $\kappa$ is increased, two of the Lyapunov exponents changes dramatically, which correspond to the Lyapunov exponents for the response system. By contrast, the other two Lyapunov exponents almost remain constant for different $\kappa$, which correspond to the Lyapunov exponents for the drive system. Generalized synchronization is achieved when the largest Lyapunov exponent of the response system becomes negative at $\kappa>0.17$.
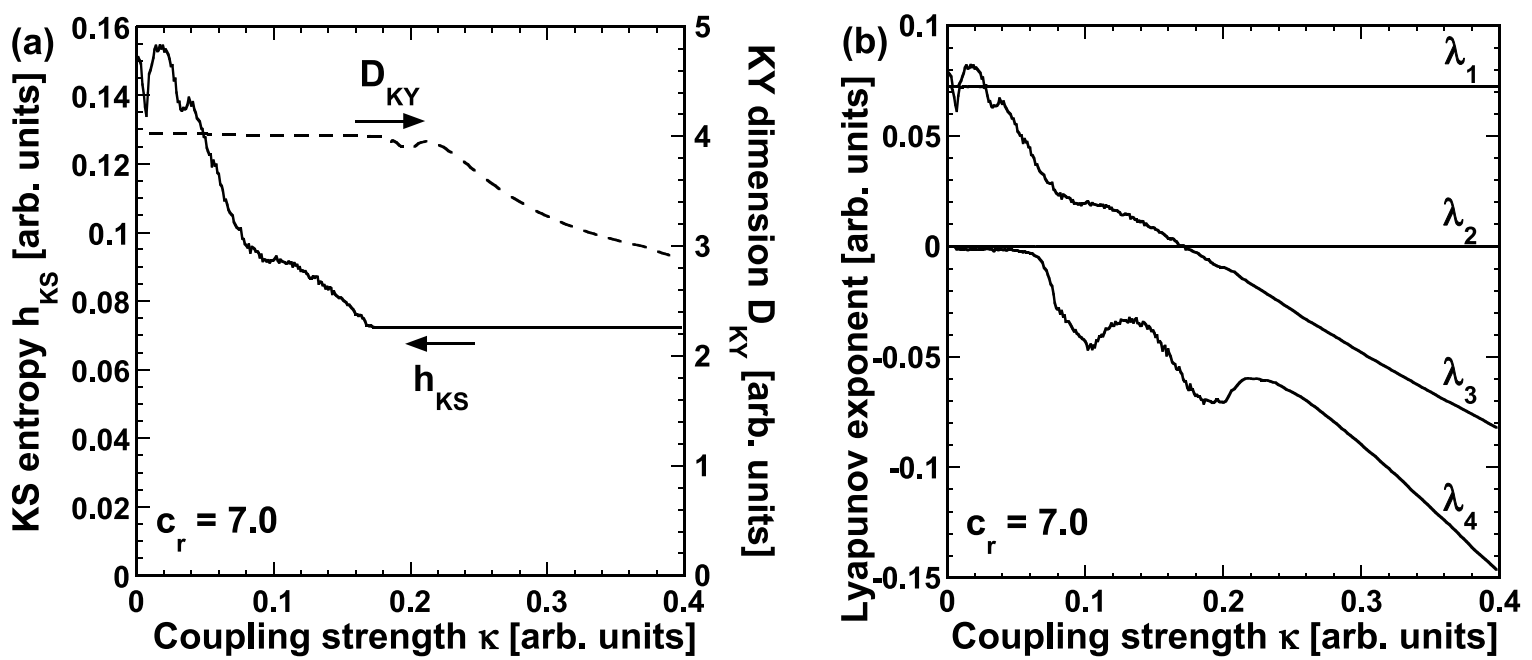

Fig. 4. (a) KS entropy $h_{K S}$ and KY dimension $D_{K Y}$, and (b) four-largest Lyapunov exponents $\left(\lambda_{1} \sim \lambda_{4}\right)$ of the coupled Rössler systems as a function of the coupling strength $\kappa$. 

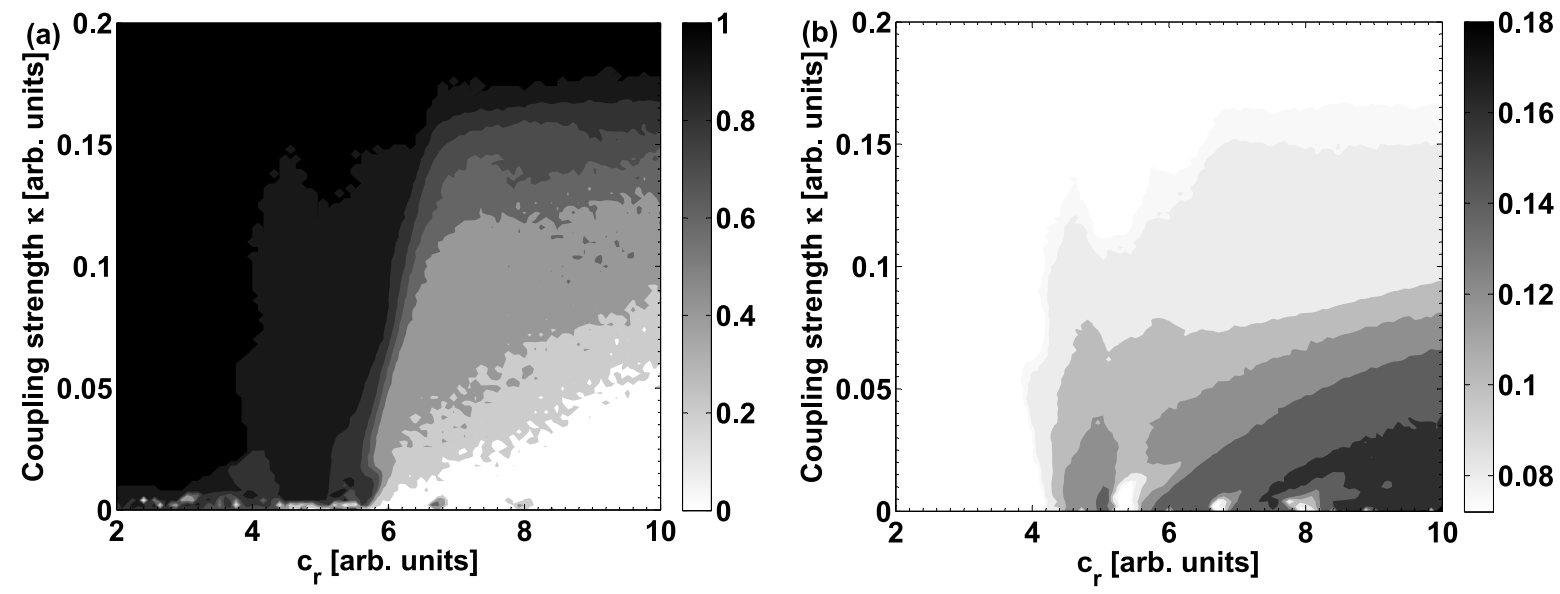

Fig. 5. Two-dimensional maps of (a) the absolute value of the crosscorrelation $C$ between the response 1 and 2 , and (b) KS entropy $h_{K S}$ of the coupled Rössler systems as functions of the coupling strength $\kappa$ and the response parameter $c_{r}\left(=c_{r 1}=c_{r 2}\right)$. (a) Black region corresponds to large cross-correlation (i.e., generalized synchronization). (b) Black region corresponds to large entropy.

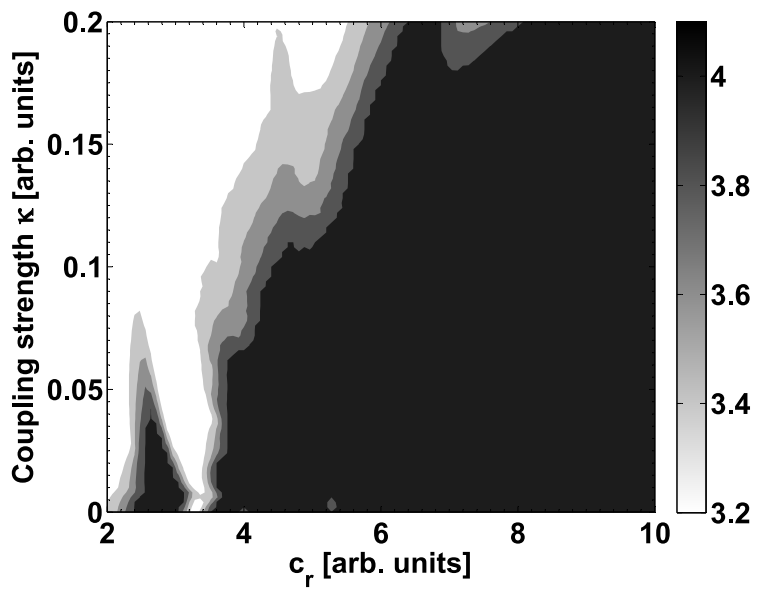

Fig. 6. Two-dimensional map of KY dimension $D_{K Y}$ of the coupled Rössler systems as functions of the coupling strength $\kappa$ and the response parameter $c_{r}\left(=c_{r 1}=c_{r 2}\right)$. Black region corresponds to large dimensionality.

Since the entropy is calculated from the sum of the positive Lyapunov exponents, the entropy decreases monotonically as $\kappa$ is increased as shown in Fig. 4(a). On the other hand, the dimensionality is almost constant outside the region of generalized synchronization, because the absolute value of $\lambda_{5}$ is much larger than those of $\lambda_{1} \sim \lambda_{4}$, and the change in $\lambda_{1} \sim \lambda_{4}$ does not affect to the calculation of the dimensionality, as described in Eq. (28). In the region of generalized synchronization, the Lyapunov exponents of $\lambda_{3}$ and $\lambda_{4}$ decrease monotonically, which affect to decrease the dimensionality.

\subsection{Two-dimensional map}

We investigate generalized synchronization and the KS entropy by changing simultaneously two parameter values of the coupling strength $\kappa$ and the response parameter value $c_{r}\left(=c_{r 1}=c_{r 2}\right)$. Figure 5 (a) shows the two-dimensional (2D) map of the cross-correlation values between the response 1 and 2 as functions of $\kappa$ and $c_{r}$. The black region indicates the achievement of generalized synchronization, while the white region corresponds to that where generalized synchronization is not achieved. Generalized synchronization is achieved for most values of $c_{r}$ for large $\kappa$, as shown in Fig. 5(a). For small $\kappa$, the region of generalized synchronization is located at small $c_{r}$. Figure $5(\mathrm{~b})$ shows the entropy $h_{K S}$ as functions of $\kappa$ and $c_{r}$, where the black region indicate large entropy. Compared Fig. 5(b) with Fig. 5(a), large entropy can be obtained at the region where generalized synchronization is not achieved, whereas small entropy is obtained at the region of generalized synchronization. It is found 

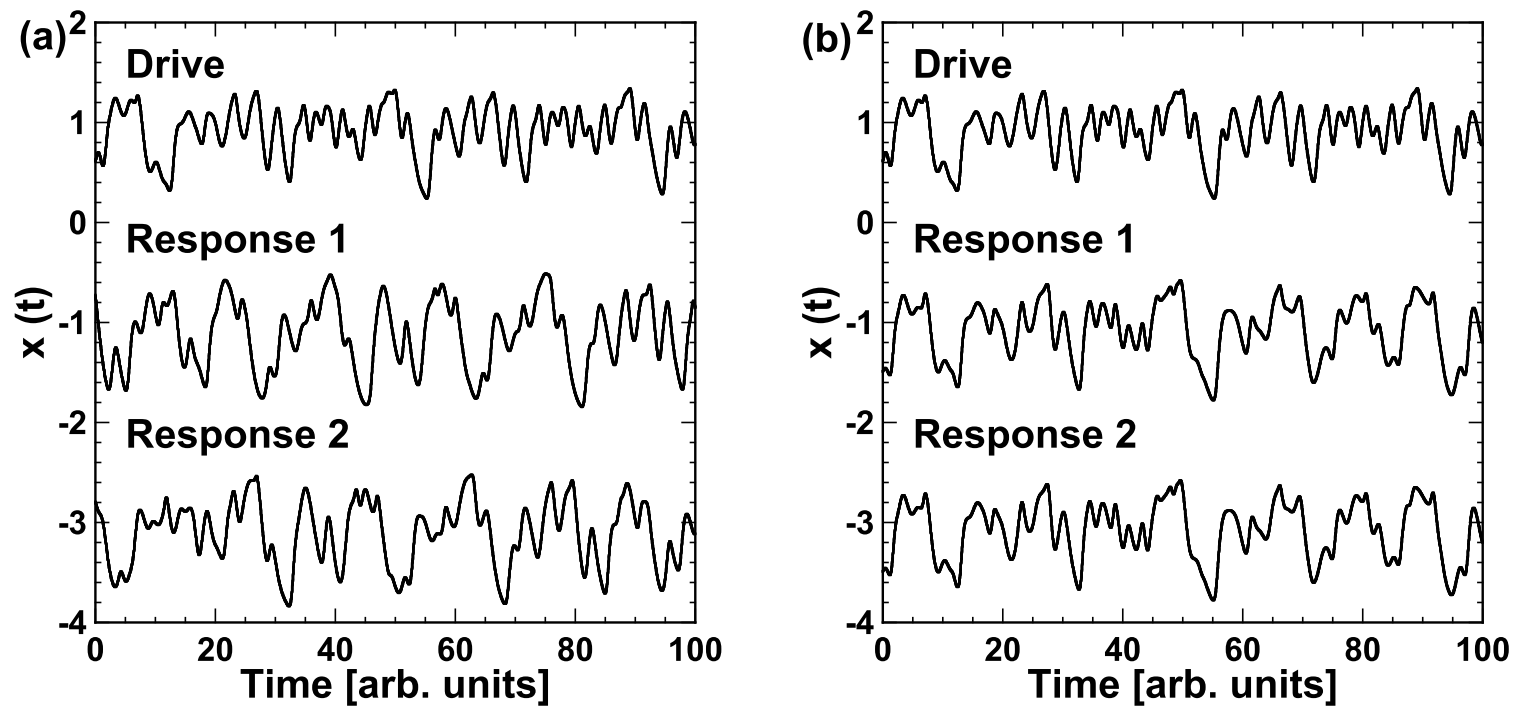

Fig. 7. Temporal waveforms of the drive, response 1 , and response 2 in the coupled Mackey-Glass systems with time-delayed feedback (a) without and (b) with coupling between the drive and response systems. The coupling strengths are (a) $\kappa=0$ and (b) $\kappa=0.8$.

that the maximum entropy almost corresponds to the sum of the entropy for the solitary drive and response systems, whereas the minimum entropy at the region of generalized synchronization equals to the entropy for the solitary drive system. This indicates that the coupling effect changes the entropy of the entire coupled system, however, the entropy ranges from the value for the solitary drive system to the value of the sum of the two solitary systems without coupling.

Figure 6 shows the 2D map of the KY dimension $D_{K Y}$ as functions of $\kappa$ and $c_{r}$. Compared Fig. 6 with Fig. 5(a), the map of $D_{K Y}$ does not directly correspond to that of generalized synchronization. $D_{K Y}$ is almost constant in the region where generalized synchronization is not achieved for small $\kappa$ and large $c_{r}$. $D_{K Y}$ decreases in the region of generalized synchronization for large $\kappa$ and small $c_{r}$. The boundaries of the changes in the region of generalized synchronization (Fig. 5(a)) and dimensionality (Fig. 6) are not well matched.

\section{Numerical results of coupled Mackey-Glass model with time-delayed feedback}

\subsection{Observation of generalized synchronization}

In this section we observe generalized synchronization in the coupled Mackey-Glass systems with timedelayed feedback. Figure 7 shows the temporal waveforms of the three coupled Mackey-Glass systems without or with coupling. In Fig. 7(a), the temporal waveforms of the response 1 and 2 have different behaviors without coupling $(\kappa=0)$. On the other hand, the temporal waveforms of the response 1 and 2 become identical at large coupling strength $(\kappa=0.8)$ and generalized synchronization can be observed as shown in Fig. 7(b). It is found that generalized synchronization can be achieved by increasing the coupling strength $\kappa$, as in the case of the coupled Rössler systems.

\subsection{Generalized synchronization and complexity}

We quantitatively evaluate the degree of generalized synchronization by using the cross-correlation function. Figure 8 shows the cross-correlation value between the response 1 and 2 as a function of the coupling strength $\kappa$. The cross correlation increases monotonically with increase of $\kappa$. Generalized synchronization is achieved at the region of $\kappa>0.6$. This result is similar to the case of the coupled Rössler systems.

To investigate the relationship between the achievement of generalized synchronization and the complexity, KS entropy $h_{K S}$ and KY dimension $D_{K Y}$ are plotted as a function of the coupling strength $\kappa$, as shown in Fig. 9(a). Without coupling $(\kappa=0)$, the value of $h_{K S}$ is 0.167 , corresponding to the 
sum of $h_{K S}$ for the solitary drive and response systems. As $\kappa$ is increased, the value of $h_{K S}$ decreases monotonically and saturates at $\kappa>0.6$, where generalized synchronization is achieved as shown in Fig. 8. The minimum value of $h_{K S}$ is 0.090 , which equals to $h_{K S}$ for the solitary drive system. These results are similar to those for the coupled Rössler systems. The dimensionality of $D_{K Y}$ also decreases monotonically even within the region of generalized synchronization as $\kappa$ is increased.

To understand the behaviors of the complexity, the Lyapunov exponents are plotted as a function of $\kappa$, as shown in Fig. 9(b). At this parameter setting, two positive Lyapunov exponents exist for each Mackey-Glass system without coupling. Therefore, two positive Lyapunov exponents are almost constant in Fig. 9(b), corresponding to the Lyapunov exponents for the drive system. On the other hand, other two positive Lyapunov exponents decrease as $\kappa$ is increased, which correspond to the Lyapunov exponents for the response system. When both of the two Lyapunov exponents for the response systems become negative at $\kappa>0.6$, generalized synchronization is achieved and the value of entropy saturates. Both KS entropy and KY dimension decrease with increase of $\kappa$ as shown in Fig. 9(a), because all the Lyapunov exponents are comparable and those for the response system decrease monotonically as shown in Fig. 9(b) in the coupled Mackey-Glass systems.

\section{Discussions}

We found the relationship between the generalized synchronization and the complexity in the coupled Rössler systems and the Mackey-Glass systems with time-delayed feedback. For both of the dynamical systems, the entropy becomes the minimum value when generalized synchronization is achieved. On the other hand, the entropy becomes the maximum value which is almost identical to the one that can be obtained when the two systems are uncoupled and independent. The former result agrees with that for optically coupled semiconductor laser systems [12], however, the latter result does not. For the optically coupled semiconductor lasers, there is another region where the entropy becomes much larger than the sum of the two solitary lasers. The region with large entropy cannot be found in both the coupled Rössler systems and the coupled Mackey-Glass systems. Therefore, the increase of entropy in optically coupled laser systems may result from the inherent characteristics of laser systems, such as fast optical-carrier interaction.

\section{Conclusions}

We have investigated the generalized synchronization and the complexity in unidirectionally coupled Rössler systems and coupled Mackey-Glass systems with time-delayed feedback. Generalized synchronization is investigated by using two identical response systems which are driven by a common drive system. To measure the complexity, KS entropy and KY dimension are calculated quantitatively

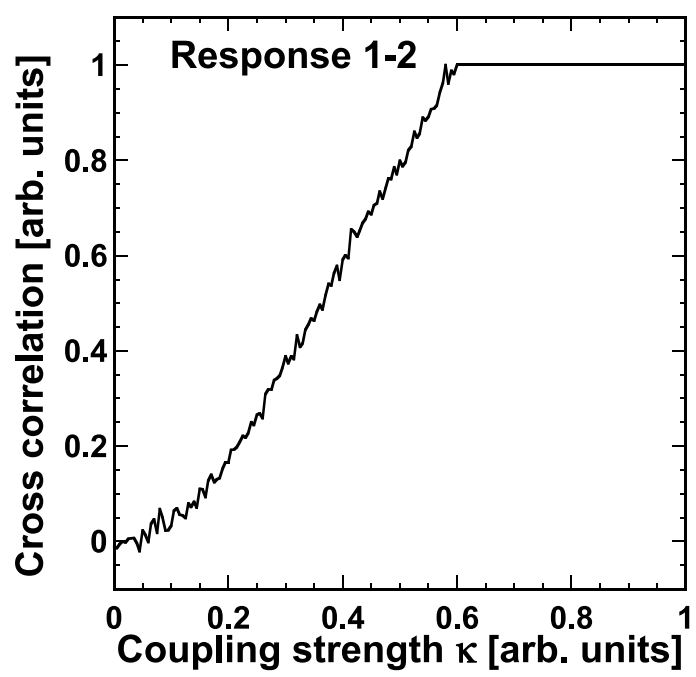

Fig. 8. Cross correlation value $C$ between the response 1 and 2 as a function of the coupling strength $\kappa$ in the coupled Mackey-Glass systems with timedelayed feedback. 

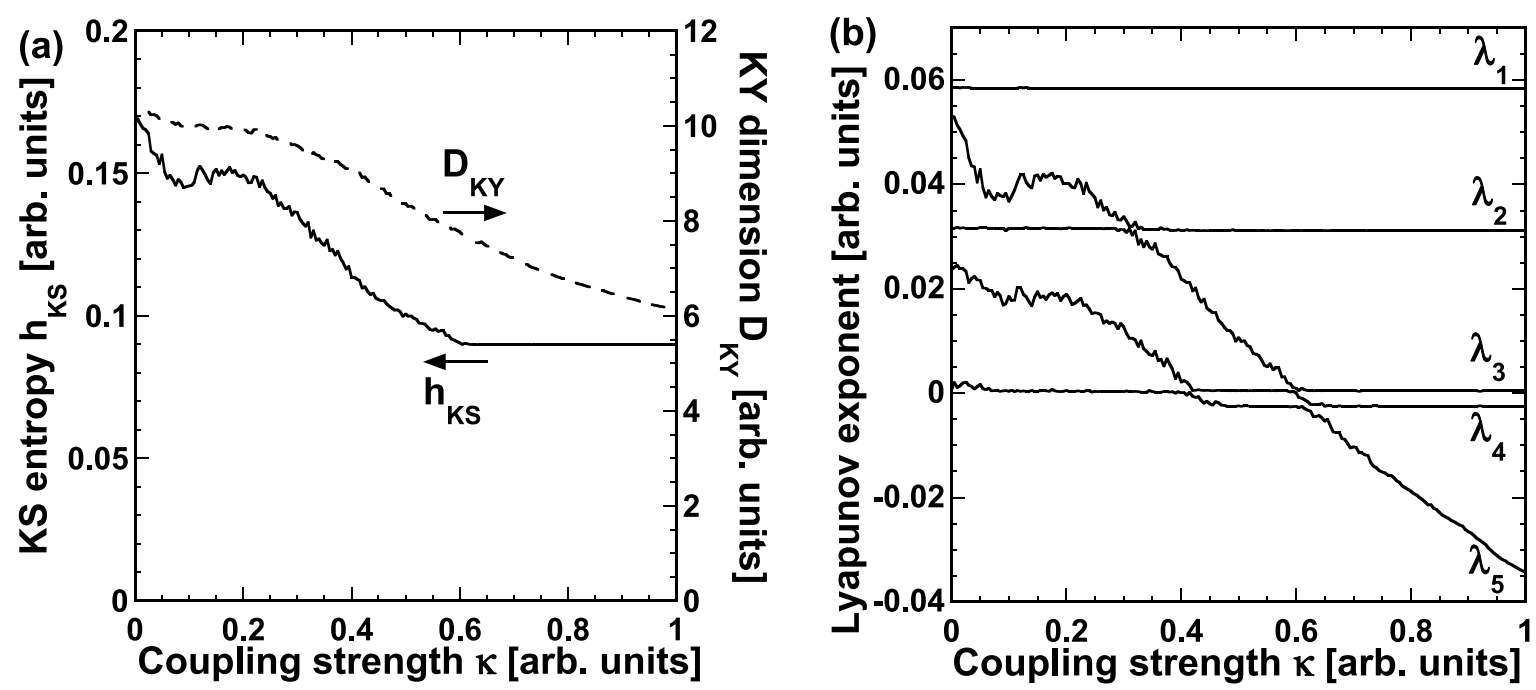

Fig. 9. (a) KS entropy $h_{K S}$ and $K Y$ dimension $D_{K Y}$, and (b) five-largest Lyapunov exponents $\left(\lambda_{1} \sim \lambda_{5}\right)$ of the coupled Mackey-Glass systems with time-delayed feedback as a function of the coupling strength $\kappa$.

from the Lyapunov spectrum by using the linearized equations. We have found that the response system shows generalized synchronization for large coupling strengths. The entropy and dimensionality decrease as the coupling strength is increased. The minimum value of entropy is obtained at the condition of generalized synchronization, whose value corresponds to the entropy for the solitary drive system, even though the temporal waveforms of the drive and response systems are significantly different. On the other hand, the maximum entropy is achieved at the region where generalized synchronization is not achieved, whose value almost corresponds to the sum of those of the two solitary drive and response systems. It is found that the coupling effect changes the entropy within these two values. By contrast, the dimensionality decreases monotonically even within the region of generalized synchronization.

\section{Acknowledgments}

We gratefully acknowledge support from Grant-in-Aid for Young Scientists from the Ministry of Education, Culture, Sports, Science and Technology in Japan, and TEPCO Research Foundation.

\section{References}

[1] H.D.I. Abarbanel, N.F. Rulkov, and M.M. Sushchik, "Generalized synchronization of chaos: The auxiliary system approach," Phys. Rev. E, vol. 53, no. 5, pp. 4528-4535, 1996.

[2] A. Pikovsky, M. Rosenblum, and J. Kurths, Synchronization, Cambridge University Press, Cambridge, England, 2001.

[3] S. Boccaletti, J. Kurths, G. Osipov, D.L. Valladares, and C.S. Zhou, "The synchronization of chaotic systems," Phys. Rep., vol. 366, p. 1, 2002.

[4] A. Uchida, R. McAllister, R. Meucci, and R. Roy, "Generalized synchronization of chaos in identical systems with hidden degrees of freedom," Phys. Rev. Lett., vol. 91, no. 17, p. 174101, 2003.

[5] A. Mukherjee, D. Ghosh, and S. Banerjee, "Synchronization of time delayed systems by common delay time modulations," Int. Scholarly Research Network ISRN Appl. Math., vol. 2011, art. ID 218458, p. 1, 2011.

[6] A. Uchida, R. McAllister, and R. Roy, "Consistency of nonlinear system response to complex drive signals," Phys. Rev. Lett., vol. 93, no. 24, p. 244102, 2004.

[7] A. Uchida, K. Yoshimura, P. Davis, S. Yoshimori, and R. Roy, "Local conditional Lyapunov exponent characterization of consistency of dynamical response of the driven Lorenz system," Phys. Rev. E, vol. 78, no. 3, p. 036203, 2008. 
[8] Z.F. Mainen and T.J. Sejnowski, "Reliability of spike timing in neocortical neurons," Science, vol. 268, no. 5216, pp. 1503-1506, 1995.

[9] T. Pérez and A. Uchida, "Reliability and synchronization in a delay-coupled neuronal network with synaptic plasticity," Phys. Rev. E, vol. 83, p. 061915, 2011.

[10] R. Pappu, B. Recht, J. Taylor, and N. Gershenfeld, "Physical one-way functions," Science, vol. 297, no. 5589, pp. 2026-2030, September 2002.

[11] R. Vicente, J. Dauden, P. Colet, and R. Toral, "Analysis and characterization of the hyperchaos generated by a semiconductor laser subject to a delayed feedback loop," IEEE J. Quantum Electron., vol. 41, no. 4, pp. 541-548, April 2005.

[12] K. Kanno and A. Uchida, "Consistency and complexity in coupled semiconductor lasers with time-delayed optical feedback," Proceedings of 2011 International Symposium on Nonlinear Theory and its Applications (NOLTA 2011), vol. 1, pp. 649-652, September 2011.

[13] O.E. Rössler, "An equation for continuous chaos," Phys. Lett. A, vol. 57, no. 5, pp. 397-398, July 1976.

[14] K. Pyragas, "Weak and strong synchronization of chaos," Phys. Rev. E, vol. 54, no. 5, p. R4508, 1996.

[15] M.C. Mackey and L. Glass, "Oscillation and chaos in physiological control systems," Science, vol. 197, no. 4300, pp. 287-289, September 1977.

[16] K. Pyragas, "Synchronization of coupled time-delay systems: Analytical estimations," Phys. Rev. E, vol. 58, no. 4, pp. 3067-3071, September 1998.

[17] J.D. Farmer, "Chaotic attractors of an infinite-dimensional dynamical system," Physica D, vol. 4, pp. 366-393, September 1982. 